\title{
Effects of Enalapril and Losartan in Left Ventricular Remodeling after Acute Myocardial Infarction: A Possible Mechanism of Prevention of Cardiac Events by Angiotensin-converting Enzyme Inhibitors and Angiotensin Receptor Blockers in High-risk Myocardial Infarction
}

\author{
Shigemasa Tani ${ }^{1,2}$, Ken Nagao ${ }^{1,2}$, Takeo Anazawa ${ }^{1,2}$, Hirofumi Kawamata ${ }^{1,2}$, Shingo Furuya ${ }^{1,2}$, \\ Hiroshi Takahashi ${ }^{1,2}$, Kiyoshi Iida ${ }^{1,2}$, Michiaki Matsumoto ${ }^{1,2}$, Narimichi Kumabe ${ }^{1,2}$, \\ Motoyuki Onikura ${ }^{1,2}$ and Atsushi Hirayama ${ }^{2}$
}

\begin{abstract}
Background Angiotensin-converting enzyme inhibitors (ACE-I) and angiotensin II receptor blockers (ARB) have been shown to have a significant cardioprotective effect in high-risk patients after myocardial infarction (MI). However, there are few data on the effects of these drugs on left-ventricular (LV) remodeling after MI in Japanese patients.

Methods and Results We randomly assigned 100 patients with anterior-wall MI who had received reperfusion therapy to treatment with either enalapril $(n=50)$ or losartan $(n=50)$, and calculated the LV ejection fraction (LVEF) and LV end-diastolic volume index (LVEDVI) in these patients at baseline and after 6 months of treatment. While a significant increase in the LVEF as compared with that at the baseline was observed in both groups, no significant difference was found in the rate of change of this parameter between the two groups. However, inverse correlations were observed between the baseline LVEF and LVEDVI and also the rates of change of the two parameters, suggesting that the greater the compromise of the LV function at baseline, the greater the preventive effect of both classes of drugs on LV remodeling.

Conclusion The results of this study suggest that neither enalapril nor losartan is superior to the other in terms of the effect on LV remodeling after MI in Japanese patients. In addition, the suppressive effect of both classes of drugs on LV remodeling was greater in patients with more extensive infarction and greater compromise of LV function at baseline.
\end{abstract}

Key words: angiotensin-converting enzyme inhibitor, angiotensin II receptor blocker, left ventricular remodeling, myocardial infarction

(Inter Med 48: 877-882, 2009)

(DOI: 10.2169/internalmedicine.48.1948)

\section{Introduction}

Large-scale randomized trials have been conducted to compare the effects of angiotensin II receptor blockers (ARBs) and angiotensin-converting enzyme inhibitors (ACE-
Is) in suppressing cardiac events in myocardial infarction (MI) patients with compromised left ventricular (LV) function; the results of these studies suggest that the cardioprotective effects of ARBs and ACE-Is are comparable $(1,2)$.

Progression of LV remodeling after MI is an important prognostic factor, and it has been shown in many clinical

${ }^{1}$ Department of Cardiology, Nihon University Surugadai Hospital, Tokyo and ${ }^{2}$ Division of Cardiovascular Medicine, Department of Medicine, Nihon University School of Medicine, Tokyo

Received for publication December 14, 2008; Accepted for publication February 19, 2009

Correspondence to Dr. Shigemasa Tani, tanishigem@yahoo.co.jp 
studies and animal experiments that both ACE-Is and ARBs suppress the progression of LV remodeling through suppression of the activity of the renin-angiotensin (RA) system (3, 4). Both ACE-Is and ARBs have been shown to exert protective effects against cardiac events in high-risk patients with compromised LV function. However, there are few data on the effects of these drugs on left ventricular (LV) remodeling after MI in Japanese patients.

The present study was undertaken to compare the effects of enalapril and losartan on LV remodeling in patients with MI and to analyze the differences in the rate/degree of LV remodeling following treatment with either of the two classes of drugs in relation to the degree of compromise of the LV function in these patients, with the goal of clarifying the effects of these drugs on LV remodeling in patients with LV dysfunction.

\section{Methods}

\section{Study subjects and protocol}

The subjects of this study were 116 patients with a first anterior-wall MI who were transported to the Critical Care Center of our hospital and received fibrinolysis and subsequent transluminal (FAST) therapy (5) between October 1997 and October 2003; patients fulfilling any of the exclusion criteria listed below were excluded from the study. The patients were randomly assigned to the losartan group or the enalapril group. Five patients dropped out of the enalapril group because of cough, and therapy was discontinued in five patients of the losartan group because of hypotension. During the six-month follow-up period, cardiac events occurred in two patients (re-infarction in one patient from the enalapril group and unstable angina in one patient from the losartan group). In four patients (enalapril group: 2, losartan group: 2) the final catheter examination results could not be analyzed. In the final analysis, therefore, 50 patients from the enalapril group and 50 patients from the losartan group were included.

Treatment with enalapril or losartan was started within 24 hours after coronary reperfusion therapy. If well-tolerated in individual cases, the drug dose was gradually increased at intervals of two weeks from the initial level of $2.5 \mathrm{mg} /$ day to a maximum of $20 \mathrm{mg} / \mathrm{day}$ in the enalapril group, and from the initial level of $12.5 \mathrm{mg}$ /day to a maximum of 100 $\mathrm{mg} /$ day in the losartan group.

The diagnosis of acute MI was based on the following criteria: persistent symptoms of acute MI plus any of the following signs on a 12-lead ECG: ST-segment elevation of at least $0.1 \mathrm{mV}$ in 2 or more of the limb leads; ST-segment elevation of at least $0.2 \mathrm{mV}$ in the precordial leads; left bundle branch block lasting longer than 30 minutes.

The exclusion criteria were: systolic blood pressure below $100 \mathrm{mmHg}$; narrowing of the left main coronary artery (luminal diameter $\geq 50 \%$ ); indications for bypass surgery; hepatic or renal dysfunction (serum creatinine $\geq 2.5 \mathrm{mg} / \mathrm{dL}$, serum alanine aminotransferase and aspartate aminotransferase $\geq 2$ times normal); history of hypersensitivity to enalapril or losartan.

The rationale and purpose of this study were disclosed to all the enrolled patients and informed consent was obtained from each patient and/or his/her family. Furthermore, adequate care was taken to protect the privacy of the individuals participating in the study. Each patient was informed of our intention not to use the clinical data from this study for purposes other than that of the present study. Only the clinical data of those individuals who gave consent for the study were included in the analyses. The study was conducted with the approval of the Ethics Committee of our institution.

\section{Coronary reperfusion therapy}

The FAST protocol employed was as follows: patients who presented to the emergency room with ischemic-type chest discomfort were evaluated by a 12-lead electrocardiogram (ECG). Intravenous coronary fibrinolysis (plus aspirin, $100-200 \mathrm{mg}$ po) was initiated immediately if the patient was deemed as an eligible candidate for FAST therapy. Patients were then assigned to receive a single bolus injection, at the optimal dose for Japanese, of either monteplase $(27.5 \mathrm{kIU} /$ $\mathrm{kg}$ ) or pamiteplase $(6.5 \mathrm{kIU} / \mathrm{kg})$. Emergency coronary angiography was performed after the fibrinolysis. If TIMI-3 flow was achieved, then urokinase $\left(500 \mathrm{U} \cdot \mathrm{kg}^{-1} \cdot \mathrm{h}^{-1}\right)$ was administered for 12 hours. If TIMI-0, -1 or -2 flow was observed in the infarct-related artery (IRA), then an adjuvant coronary intervention procedure was performed immediately.

The adjuvant intervention was selected according to whether or not there was clear evidence of thrombosis (i.e., contrast medium flowing into the thrombus); in the presence of clear evidence of thrombosis, intracoronary fibrinolysis using pro-urokinase at the dose of up to 3,000 units was selected; in the absence of such evidence, percutaneous transluminal coronary angioplasty was selected.

The contraindications for thrombolytic therapy in the patients were as follows: more than 6 hours after the onset of acute MI; age over 75 years; history of stroke or central nervous system damage; left main coronary artery disease; active bleeding or bleeding tendency; recent trauma or major surgery; systolic blood pressure greater than $180 \mathrm{mmHg}$ or diastolic blood pressure greater than $110 \mathrm{mmHg}$ as measured in the emergency room.

\section{Measurements of left ventricular volume and function}

As indicators of LV remodeling, the left ventricular ejection fraction (LVEF) and left ventricular end-diastolic volume index (LVEDVI) were calculated from left ventriculograms (LVG) obtained in the acute phase (baseline) and at 6 months after the occurrence of MI. Two cardiologists who were unaware of the treatment assignment (enalapril or losartan) analyzed the LVGs. Ventricular silhouettes in the right anterior oblique projection were digitized with an LVG analysis system, and the LVEF and LVEDVI were deter- 
mined.

\section{Data collection}

The initial prospective data were collected from our coronary reperfusion therapy database and the medical records in our hospital. Each coronary lesion that resulted in a reduction of the luminal diameter by more than $50 \%$ was considered as clinically significant. Cohn's grade 3 signified good development of collateral vessels. Successful reperfusion of the IRA was considered successful if TIMI-3 flow was achieved. Door-to-TIMI-3 time was defined as the timeinterval from arrival at the emergency room until achievement of TIMI-3 flow. Drugs that were prescribed at discharge were considered as background factors: aspirin, $\beta$ blockers, calcium channel blockers, statins, nitrates, spironolactone.

\section{Statistical analysis}

Data were expressed as mean \pm SD and as percentages. For category comparison between the two groups, the chisquare test was used. Unpaired two-group comparison was used for comparison of continuous variables between the two groups. The paired t-test was employed for comparison of the LVEF between the baseline and at the 6 months follow-up in each group. To evaluate the degree of suppression of LV remodeling in relation to the baseline LV function, the correlations of the baseline LVEF and LVEDVI to the percent change in the respective parameters were calculated. All of the statistical analyses were performed with the SPSS software (version 12.0.1, SPSS Inc.). P values of less than 0.05 were considered to be statistically significant.

\section{Results}

Table 1 shows the background variables of the patients included in the analyses. The mean dose level of enalapril was $12.5 \pm 5.3 \mathrm{mg} /$ day, and that of losartan was $60.4 \pm 12 \mathrm{mg} /$ day.

There were no significant differences in the systolic or diastolic blood pressure between the enalapril group and losartan group either at the time of enrolment in the study (enalapril group vs. losartan group: $132 \pm 12$ vs. $133 \pm 11$ mmHg; $\mathrm{p}=0.87)$, or at one month $(122 \pm 12$ vs. $123 \pm 11$ $\mathrm{mmHg}$; $\mathrm{p}=0.74), 3$ months $(123 \pm 12$ vs. $118 \pm 11 \mathrm{mmHg}$; $=$ $0.89)$ or 6 months after enrolment in the study (112 \pm 12 vs., $110 \pm 11 \mathrm{mmHg}$; $\mathrm{p}=0.85)$.

\section{Changes in the LVEF and LVEDVI and comparison of the changes between the two groups}

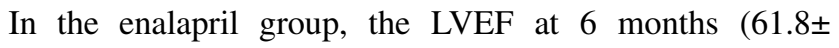
$12.3 \%$ ) was significantly higher than that at the baseline $(53.5 \pm 11.3 \%)(\mathrm{p}<0.0001)$. Likewise, in the losartan group also, the LVEF at 6 months $(60.9 \pm 10.1 \%)$ was significantly higher than that at the baseline $(53.0 \pm 10.7 \%) \quad(p=0.0004)$. No significant inter-group difference was noted in the percent change of the LVEF between the two groups (enalapril group vs. losartan group: $16.6 \pm 18.7 \%$ vs. $16.5 \pm 17.3 \%$; $p=$
0.97).

In the enalapril group, the LVEDVI at 6 months $(81.4 \pm$ $21.3 \mathrm{~L} / \mathrm{m}^{2}$ ) was significantly lower than that at the baseline $\left(101.3 \pm 28.3 \mathrm{~L} / \mathrm{m}^{2}\right) \quad(\mathrm{p}<0.0001)$. Similarly, in the losartan group also, the LVEDVI at 6 months $\left(79.9 \pm 21.3 \mathrm{~L} / \mathrm{m}^{2}\right)$ was significantly lower than that at the baseline $(98.1 \pm 30.1$ $\left.\mathrm{L} / \mathrm{m}^{2}\right)(\mathrm{p}<0.0001)$. There was no significant difference in the percent change of the LVEDVI between the two groups (enalapril group vs. losartan group: $-15.2 \pm 32.4 \%$ vs. $-13.7 \pm$ $24.5 \% ; \mathrm{p}=0.85)$.

\section{Correlation between the baseline $L V$ function and the changes in the $L V$ function after 6 months of treatment in the two groups}

In the entire population, the baseline LVEF was negatively correlated with the percent change of this parameter observed at 6 months after enrolment in the study $(\mathrm{R}=-$ $0.502, \mathrm{p}<0.0001)$. A negative correlation between the baseline LVEF and the percent change of this parameter at 6 months was also observed in both groups analyzed individually (enalapril group: $\mathrm{R}=-0.517, \mathrm{p}=0.0001$, losartan group: $\mathrm{R}=-0.486, \mathrm{p}=0.0003$ ) (Fig. 1).

In the entire population, the baseline LVEDVI was negatively correlated with the percent change of this parameter observed at 6 months after enrolment in the study $(\mathrm{R}=-0.677, \mathrm{p}<0.0001)$. A negative correlation between the baseline LVEDVI and the percent change of this parameter at 6 months was also observed in both groups analyzed individually (enalapril group: $\mathrm{R}=-0.701, \mathrm{p}<0.0001$, losartan group: $\mathrm{R}=-0.657, \mathrm{p}<0.0001$ ) (Fig. 2).

Thus, in both the enalapril and losartan groups, the percent improvement in LV remodeling after 6 months of treatment, as assessed by the two parameters mentioned above, was higher in the patients with a poorer baseline LV function, and less marked in those with relatively well-preserved LV function (less extensive and/or severe infarction) at baseline. These results suggest that high-risk patients with more extensive infarction are more likely to accrue the beneficial effects of the two classes of drugs examined.

\section{Discussion}

The present study had important clinical findings. There was no significant difference in improvement of LV remodeling between the enalapril group and the losartan group, that is, both drugs suppressed LV remodeling to an equivalent degree. It is noteworthy, however, that the effect of both enalapril and losartan in suppressing LV remodeling was greater in patients with more extensive infarction and lower baseline LV function levels.

To date, two large-scale randomized clinical studies have been carried out to compare the usefulness of ACE-Is and ARBs for secondary prevention of MI: OPTIMAAL (1) and VALIANT (2). Neither of these revealed any statistically significant difference in the incidence of death from heart failure between patients treated with ACE-Is and those 
Table 1. Patient Characteristics

\begin{tabular}{|c|c|c|c|c|}
\hline & $\begin{array}{c}\text { All cases } \\
(n=100)\end{array}$ & $\begin{array}{l}\text { Enalapril group } \\
\qquad(n=50)\end{array}$ & $\begin{array}{c}\text { Losartan group } \\
(n=50)\end{array}$ & p value \\
\hline \multicolumn{5}{|l|}{ General } \\
\hline Age (years) & $61 \pm 7.0$ & $61 \pm 6.7$ & $60 \pm 7.2$ & 0.41 \\
\hline Male, n (\%) & $57(86)$ & $28(85)$ & $29(88)$ & 0.72 \\
\hline BMI $\left(\mathrm{kg} / \mathrm{m}^{2}\right)$ & $24 \pm 2.0$ & $25 \pm 1.9$ & $24 \pm 2.0$ & 0.66 \\
\hline \multicolumn{5}{|l|}{ Cardiovascular stature } \\
\hline Multi vessel disease, n (\%) & $29(29)$ & $21(42)$ & $23(45)$ & 0.80 \\
\hline Culprit lesion (proximal site of LAD) & $29(29)$ & $14(27)$ & $15(30)$ & 0.79 \\
\hline Peak CK (IU/L) & $3332 \pm 1292$ & $3339 \pm 1238$ & $3321 \pm 1366$ & 0.80 \\
\hline Peak CK MB (IU/L) & $304 \pm 134$ & $302 \pm 135$ & $306 \pm 136$ & 0.89 \\
\hline Killip class I, n (\%) & $76(76)$ & $37(73)$ & $40(79)$ & 0.57 \\
\hline Preinfarction angina, $\mathrm{n}(\%)$ & $47(47)$ & $26(45)$ & $24(48)$ & 0.80 \\
\hline Good collateral, $\mathrm{n}(\%)$ & $29(29$ & $14(27)$ & $15(30)$ & 0.79 \\
\hline Successful reperfusion, $\mathrm{n}(\%)$ & $100(100)$ & $100(100)$ & $50(100)$ & - \\
\hline Door-to-TIMI 3 time (minutes) & $95 \pm 69$ & $94 \pm 67$ & $97 \pm 72$ & 0.86 \\
\hline Patency rate of IRA at 6 months, n (\%) & $100(100)$ & $50(100)$ & $50(100)$ & - \\
\hline \multicolumn{5}{|l|}{ Risk factors } \\
\hline Hypertension, n (\%) & $100(100)$ & $24(48)$ & $26(52)$ & 0.80 \\
\hline Diabtes mellitus, n (\%) & $23(23)$ & $12(24)$ & $11(21)$ & 0.77 \\
\hline Hyperlipidemia, n (\%) & $54(54)$ & $28(55)$ & $28(55)$ & 0.80 \\
\hline Cigarette smoking, $\mathrm{n}(\%)$ & $55(55)$ & $17(52)$ & $19(58)$ & 0.80 \\
\hline \multicolumn{5}{|l|}{$\begin{array}{l}\text { Cigarette smoking, } \mathrm{n}(\%) \\
\text { Medication use }\end{array}$} \\
\hline Aspirin, n (\%) & $100(100)$ & $50(100)$ & $50(100)$ & 1.0 \\
\hline$\beta$ blockers, $\mathrm{n}(\%)$ & $29(29)$ & $14(27)$ & $15(30)$ & 0.79 \\
\hline Calcium channel blockers, $\mathrm{n}(\%)$ & $23(23)$ & $11(21)$ & $12(24)$ & 0.77 \\
\hline Statins, $\mathrm{n}(\%)$ & $70(70)$ & $34(67)$ & $34(73)$ & 0.59 \\
\hline Nitrates, n (\%) & $17(17)$ & $8(15)$ & $9(18)$ & 0.74 \\
\hline Spironolactone, n (\%) & $17(17)$ & $8(15)$ & $9(18)$ & 0.74 \\
\hline \multicolumn{5}{|l|}{$\begin{array}{l}\text { Spironolactone, } \mathrm{n}(\%) \\
\text { Fibrinolytic agent }\end{array}$} \\
\hline Monteplase / Pamiteplase, n (\%) & $50(50) / 50(50)$ & $24(48) / 26(52)$ & $26(52) / 24(48)$ & 0.84 \\
\hline Rescue PCI, n (\%) & $30(30)$ & $14(27)$ & $17(33)$ & 0.59 \\
\hline
\end{tabular}

BMI: body mass index; Proximal site of LAD indicates segment 6 (America Heart Association clasissfication); LAD: left anterior descending; CK: creatin

phosphokinase; TIMI: thrombolysis in myocardial infarction; IRA: infarct-related artery; PCI: percutaneous coronary intervention

treated with ARBs. In other words, neither of the studies revealed any superior efficacy of ACE-Is over ARBs. In the present study also, the two drugs were found to exert equivalent efficacy in suppressing LV remodeling, endorsing the results from the two large-scale studies mentioned above.

It has been reported that ACE-Is exert cardiovascular protective effects through suppression of several mechanisms by stimulating the RA system. However, when used for secondary prevention of MI, ACE-Is are expected to exert a cardioprotective effect mainly by suppressing LV remodeling (6). This view is endorsed by the finding from a large-scale clinical study that the effect of ACE-Is in suppressing cardiac events was more marked in high-risk patients with poorer LV function levels. In regard to ARBs, it has been shown in shown in two large-scale studies, i.e., OPTIMAAL and VALLIANT, that their effect in preventing cardiac events are comparable to that of captopril. Therefore, largescale clinical studies carried out to evaluate the effects in secondary prevention of MI have also revealed that ARBs and ACE-Is exert relatively greater efficacy in suppressing LV remodeling in high-risk patients with poorer LV function at baseline.

The CHARM (7), in which the effects of candesartan in patients not limited to those with myocardial infarction were studied, the effect of the drug in preventing cardiac events was greater in patients with a lower LVEF at baseline. In the present study, suppression of LV remodeling was more marked in patients with poorer LV function in both the enalapril group and the losartan group, endorsing the findings from the aforementioned large-scale clinical studies, that the beneficial effects of both ARBs and ACE-Is in preventing cardiac events were more pronounced in higher risk patients with poorer LV function.

Regarding the optimal timing of use of ACE-Is, the results of large scale trials (8-10) suggest that early treatment is the most useful. It has been suggested that treatment with these drugs is ideally started within 24 hours of the onset of MI, i.e., immediately after stabilization of the hemodynamics following completion of coronary reperfusion therapy, with the drug started at a low initial dose level, the dose being gradually increased thereafter. In previous large-scale clinical studies conducted to evaluate the effects of ARBs, the drug was not administered during the very early stage after the onset of MI; that is, treatment was started a median of 3 days after the onset of MI in the OPTIMAAL and mean of 4.9 days after the onset of MI in the VALIANT. In the present study, on the other hand, the losartan administered during the early stage after the onset of MI (within 24 hours of coronary reperfusion therapy), suppressed LV remodeling to a degree equivalent to that achieved with enalapril treatment. This result suggests that suppression of the RA system during the early stage after the onset of MI allows effective suppression of LV remodeling. 

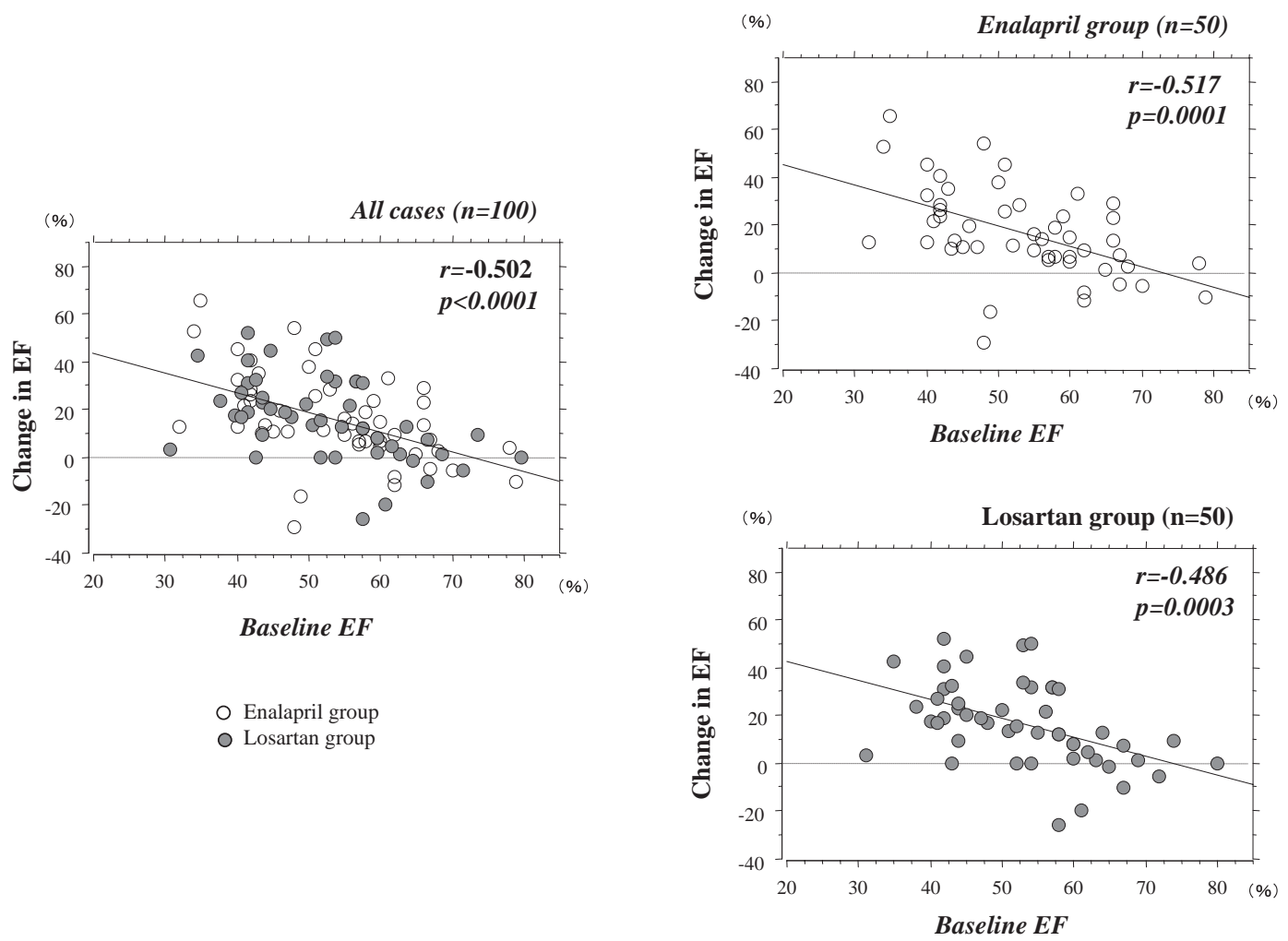

Figure 1. Correlation between the baseline EF and the change in EF.
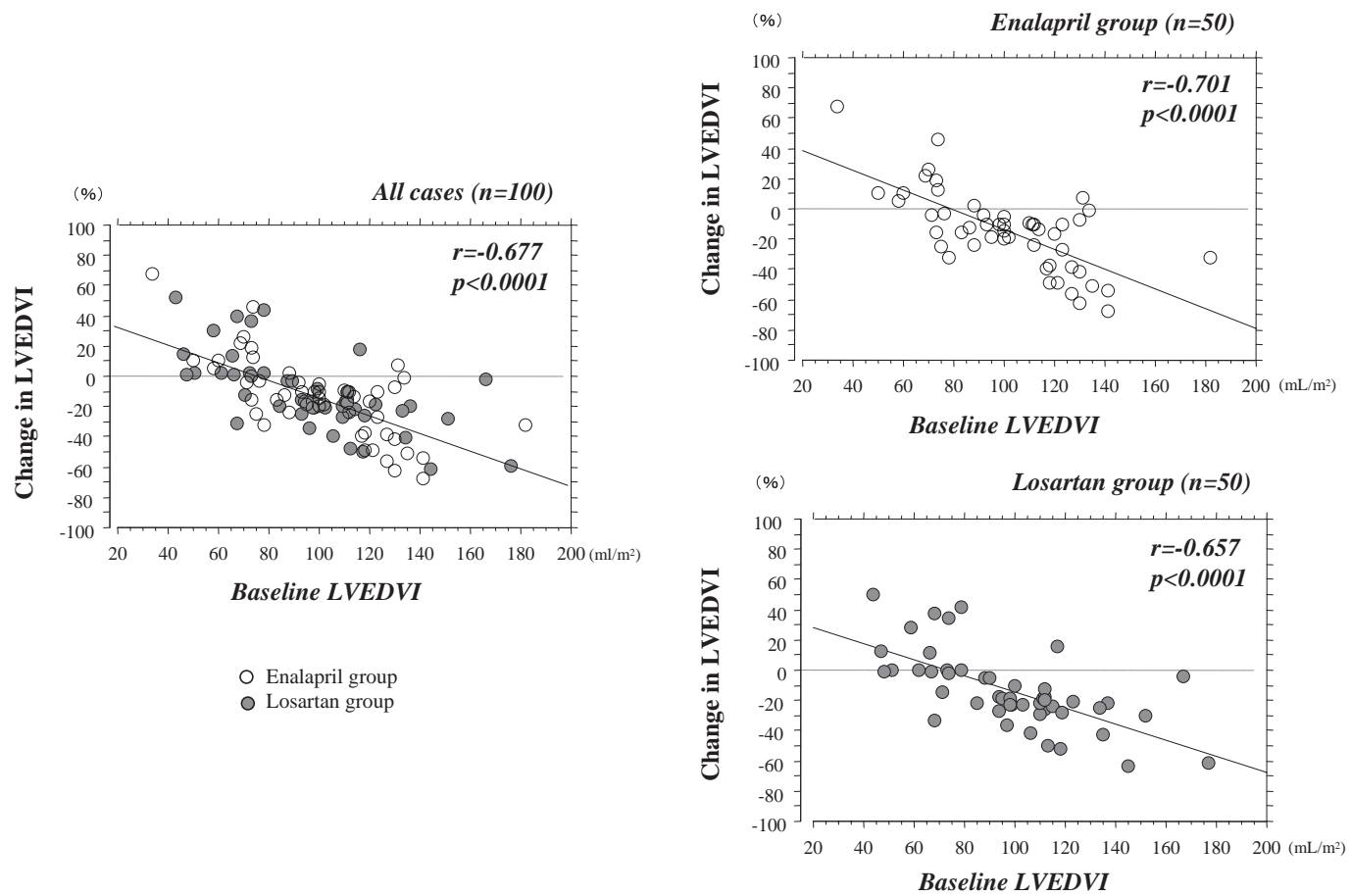

Figure 2. Correlation between baseline LVEDVI and the change in LVEDVI.

None of these studied revealed any significant difference in the efficacy of ACE-Is and ARBs in preventing the progression to heart failure. Thus, there seems to be a consensus at present that both ACE-Is and ARBs are closely involved in preventing the progression of LV remodeling and that they do not differ from each other in terms of their effi- cacy in preventing the progression of heart failure. In the VALLIANT sub study evaluated with echocardiogram, treatment with captopril and valsartan in patients with MI complicated by LV dysfunction was associated with similar changes in LV size and function in the 20 months (11). Some small sample studies have revealed inconsistent effects 
regarding the superiority of one over the other of enalapril and losartan in terms of their efficacy in suppressing LV remodeling after MI $(4,12)$. We therefore believe that future studies might shed new light on this subject. ARBs are known to have class effects, such as high affinity for the AT 1 receptor, modification of the drug half-life and hypotensive actions (13). The evidence collected on the efficacy as a secondary prophylactic agent in cases of $\mathrm{MI}$ is less adequate for ARBs than for ACE-Is.

\section{Study limitations}

The present study had several limitations. In the present study, we did not incorporate a control group not treated with enalapril or losartan, since we thought it was ethically inappropriate to avoid the use of these drugs in MI patients with decreased LV function. Accordingly, it was difficult to rule out the effects of confounding factors on LV remodel- ing (e.g., recovery from stunning while evaluating improvement of LV function). Finally, it might be desirable to use a humoral factor for assessment in this kind of study.

\section{Conclusion}

In patients with anterior MI who received early coronary reperfusion therapy, no significant difference was observed between enalapril and losartan in terms of their efficacy in suppressing LV remodeling. However, both drugs yielded more marked improvement of $\mathrm{LV}$ remodeling in patients with poorer LV function at baseline. This finding seems to serve as an important basis for evaluation of the usefulness of ACE-Is and ARBs in the secondary prevention of MI in Japanese patients. However, it would be desirable to conduct studies including a greater number of subjects to collect further evidence.

\section{References}

1. Dickstein K, Kjekshus J; The OPTIMAAL Steering Committee, for the OPTIMAAL Study Group. Effects of losartan and captopril on mortality and morbidity in high-risk patients after acute myocardial infarction: the OPTIMAAL randomised trial. Optimal Trial in Myocardial Infarction with Angiotensin II Antagonist Losartan. Lancet 360: 752-760, 2002.

2. Pfeffer MA, McMurray JJ, Velazquez EJ; Valsartan in Acute Myocardial Infarction Trial Investigators. Valsartan, captopril, or both in myocardial infarction complicated by heart failure, left ventricular dysfunction, or both. N Engl J Med 349: 1893-1906, 2003.

3. Kim KS, Yoshiyama M, Izumi Y, et al. Effects of combination of ACE inhibitorand angiotensin receptor blocker on cardiac remodeling, cardiac function, and survival in rat heart failure. Circulation 103: 148-154, 2001.

4. Maia LN, Nicolau JC, Vítola JV, et al. Prospective evaluation comparing the effects of enalapril and losartan in left ventricular remodeling after acute myocardial infarction. Am Heart J 145: E 21, 2003.

5. Nagao K, Hayashi N, Kanmatsuse K, Kikuchi S, Ohuba T, Takahashi H; FAST Trial. An early and complete reperfusion strategy for acute myocardial infarction using fibrinolysis and subsequent transluminal therapy--The FAST trial. Circ J 66: 576-582, 2002.

6. Schmieder RE, Hilgers KF, Schlaich MP, Schmidt BM. Reninangiotensin system and cardiovascular risk. Lancet 369: 12081219, 2007.

7. Young JB, Dunlap ME, Pfeffer MA, et al; Candesartan in Heart failure Assessment of Reduction in Mortality and morbidity (CHARM) Investigators and Committees. Mortality and morbidity reduction with Candesartan in patients with chronic heart failure and left ventricular systolic dysfunction: results of the CHARM low-left ventricular ejection fraction trials. Circulation 110: 26182626, 2004.

8. Pfeffer MA, Braunwald E, Moyé LA, et al. Effect of captopril on mortality and morbidity in patients with left ventricular dysfunction after myocardial infarction. Results of the survival and ventricular enlargement trial. The SAVE Investigators $\mathrm{N}$ Engl J Med 327: 669-677, 1992.

9. ISIS-4 (Fourth International Study of Infarct Survival) Collaborative Group ISIS-4: a randomised factorial trial assessing early oral captopril, oral mononitrate, and intravenous magnesium sulphate in 58,050 patients with suspected acute myocardial infarction. Lancet 345: 669-685, 1995.

10. GISSI-3 effects of lisinopril and transdermal glyceryl trinitrate singly and together on 6-week mortality and ventricular function after acute myocardial infarction. Gruppo Italiano per lo Studio della Sopravvivenza nell'infarto Miocardico. Lancet 343: 11151122, 1994.

11. Solomon SD, Skali H, Anavekar NS, et al. Changes in ventricular size and function in patients treated with valsartan, captopril, or both after myocardial infarction. Circulation 111: 3411-3419, 2005.

12. Onodera H, Matsunaga T, Tamura $Y$, et al. Enalapril suppresses ventricular remodeling more effectively than losartan in patients with acute myocardial infarction. Am Heart J 150: 689, 2005.

13. Ssraili ZH. Clinical pharmacokinetics of angiotensin II (AT1) receptor blockers in hypertension. J Hum Hypertens 14 Suppl 1: S 73-86, 2000.

(C) 2009 The Japanese Society of Internal Medicine http://www.naika.or.jp/imindex.html 